\title{
Spatiotemporal Water Quality Assessment of Dumate Al-Jandal Lake (KSA) Using Environmetric Techniques
}

\author{
Mohammed Sharif Al-Sheraideh \\ *Imam Abdurrahman Bin Faisal University KSA, College of Science, Chemistry Department-Kingdom of Saudi \\ Arabia -B.O. 383 Dammam -31113, \\ msalsheraideh@uod.edu.sa
}

\begin{abstract}
Environmentally, the objective of the study conducted in evaluating the spatiotemporal water quality assessment using some statistical techniques. Physicochemical characteristics determination applied on Dumate al-Jandal Lake, whether the analysis of water quality is good for agricultural irrigation or other ecosystem services. Sampling and measurements were taken weekly at five sites started from February 2009 to January 2011. Descriptive analysis as well as the $95 \%$ confidence intervals, Wilks' Lambda Statistics, MANOVA and ANOVA showed no presence of significant difference at the level of $p<0.05$ among seasons and between sites, except the mean effect only for some parameter like Iron on the physiochemical parameters whereas a significant difference among the mean of physiochemical characteristics of water data to sites for the parameters like pH and nitrate, while there are no differences among the mean of physiochemical characteristics of water data of sites for the other parameters. Multiple comparison t-test shows the differences between means Sites of $(1,3)$ and $(2,5)$ as well as between (3) and (5) for the parameter $\mathrm{pH}$. Results showed a significant difference at $(\mathrm{p}<$ 0.05 ) between the means of Site (1) and all Sites (3,4 and 5) as well as between Site (2) and (3) for nitrate, but a significant difference at $(p<0.05)$ among the mean associated with seasons for temperature, electrical conductivity, specific density, sulphate, nitrite, ammonia, chloride, total hardness, total alkalinity, manganese, magnesium and calcium hardness, while there are no differences among the mean of seasons for the other parameters. Whereas results of multiple comparison $t$-test showed that a differences between the means of season $(i)$ and season $(j)$, for each parameter. The study concluded that some of physicochemical parameters were reflected the presence of pollutants and absence of good ecosystem activities. To avoid seasonal pollution, water lake management are recommended.
\end{abstract}

Keywords: Physicochemical analysis, Aquatic ecosystem, Environmetric Techniques.

\section{Introduction}

Dumate al-Jandal has a large lake, located about $6 \mathrm{~km}$ away western town Dumate Al-Jundal in Al-Jouf Province of Saudi Arabia. The storage capacity of the lake is about 10 million cubic meters of water and annually evaporates the same amount of nutrition. Its surface area is estimated of about one million one hundred thousand square meters with about 8 kilometers varying depth and approximately 1.10 million $\mathrm{m}^{2}$ and lake level is $643 \mathrm{~m}$ which is about $585 \mathrm{~m}$ above the sea level. Its surface area is about $8 \mathrm{~km}$ with various depths. From northern reservoir, the water pumps through large pipes directly to the lake so weathering changes besides linkages seasonal variation in precipitation, surface runoff, interflow have a strong effect on the concentration of pollutants which affect lake water quality [1]. Due lack of good water quality information about the lake, protection of its water resources becomes topmost priority. In this study spatiotemporal analysis and Researchwise, one of the important stages in the protection and conservation of these resources of water and quality of the aquatic ecosystems [2]. In general, water quality is strongly related with physical, chemical and biological characteristics which are determined the healthy status of any aquatic ecosystem [3]. Therefore, quality of water assessment in any ecosystem requires significant information about the available resources for supporting ecosystem life [4]. Because the physicochemical characteristics of water resources are affected their species composition, productivities and abundance, therefore, this study was conducted to understand the physicochemical properties of the artificial lake for different types of ecosystem services and activities for one year from February 2009 to January 2011 [5]. 
The statistical measures of environmental data make spatiotemporal variations of water quality often difficult to interpret, so statistical approaches like descriptive of minimum, maximum, mean and standard deviation values as well as the $95 \%$ confidence intervals are used in this study for providing a reliable analysis to summarize these data for each parameter to all sites and seasons [6]. Also, Wilks' Lambda Statistics in the Multivariate Analysis of Variance (MANOVA) techniques have been widely used as unbiased methods in analysis of water quality and for evaluating sites and seasons which have mean effect parameter on the physiochemical of water [7]. One Way Analysis of Variance (ANOVA) and the results of multiple comparison t-test techniques will be used to show if there are differences among the mean of physiochemical characteristics of water data of sites that drawing out meaningful conclusions [8].

Physical parameters such as temperature, acidity, turbidity and others have known to affect in ecosystem as result of the interaction of both physical and chemical properties of water which plays a significant role in the composition. Also, it is a great environmental concern since it one of the major available water resources for animal and agricultural irrigation consumption. Until now no previously available data on the chemical, physical properties reported on the lake, which is the primary purpose of this study to fill this gap [9]. The major objective of this research study focusing into the relationship between the results of 22 parameters and their spatiotemporal environmental variations which determining water quality productivity and a new methodology for examining the impact of all the sources of pollution in the lake and to identify the parameters responsible for spatiotemporal variability in water quality using.

\section{Materials and Methods}

\subsection{Study Area}

The present study was carried out on the largest and only artificial lake in Dumate Al-Jandal city, that located in the northern part of the Arabian Peninsula in Saudi Arabia, that fed from the surplus of agricultural irrigation water through northern and southern major reservoirs in the city, which is one of the richest regions of the kingdom with ground water that pumped to it. Water samples were taken from surface of different locations in the lake as seen in Fig. 1 and Fig. 2.

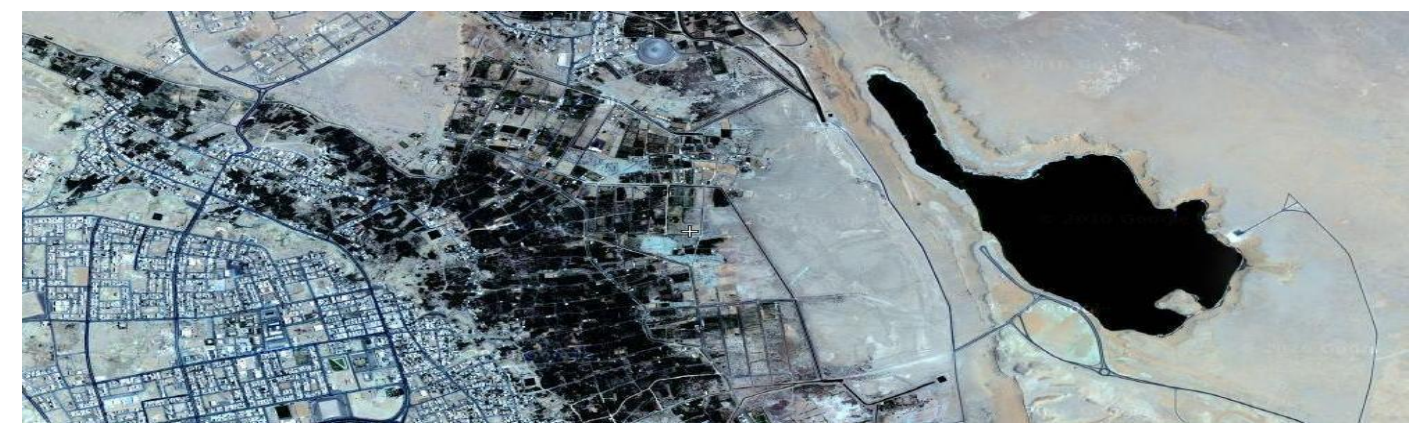

Figure 1: Satellite image of lake Dumate Al-Jundal -KSA.

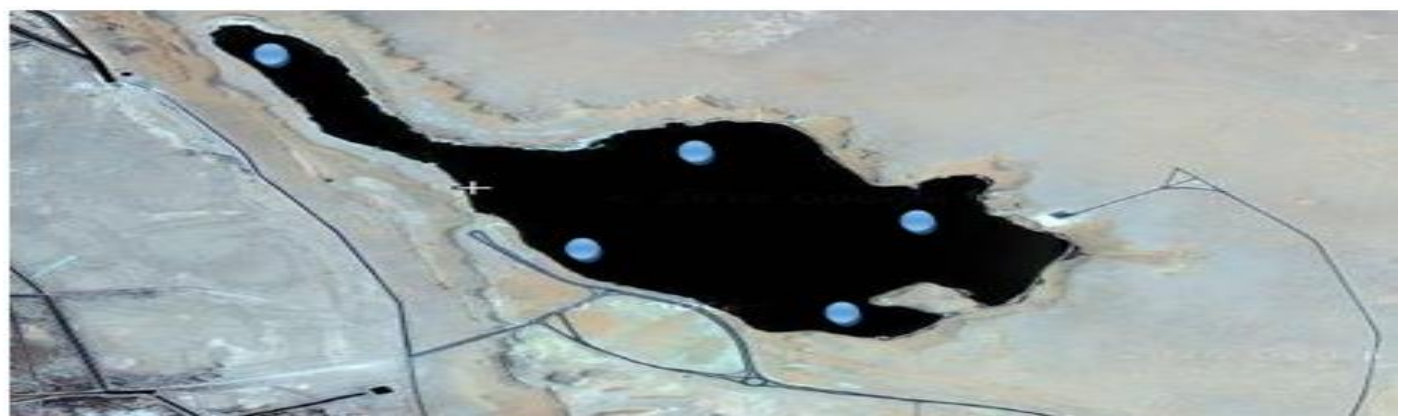

Figure 2: Location of the study monitoring stations area in the Dumate Al-Jundal lake-KSA 


\subsection{Sampling and Analysis}

Water samples were collected weekly from five sampling sites between 11.00 and 1.00 O'clock a day, from February 2009 to January 2010. Then water samples collected from each site placed in one-liter polyethylene pre-rinsed with acid, filtered with a $0.45 \mu \mathrm{m}$ membrane using vacuum filtration apparatus and add a few drops of $36 \% \mathrm{HCl}$ to keep pH less than two and refrigerated at $4{ }^{\circ} \mathrm{C}$ for the laboratory analysis. Physical parameters like temperature, conductivity and $\mathrm{pH}$, were determined respectively on the spot using a thermometer $( \pm 0.10$ ${ }^{\circ} \mathrm{C}$ ), $\mathrm{pH}$ meter (model HANNA), and an electric conductivity meter (model Bischof L17). While other chemical parameters such as total alkalinity, total hardness, calcium, magnesium, sulfate, nitrite, nitrate, ammonia and phosphate were determined in the laboratory within 24 - 48 hours of sampling according to standard methods of Parsons et al. (1984) and Golterman and Clymo (1969) and APHA (1998) [10-11].

\subsection{Statistical Analysis}

To simplify studying seasonal effect on water lake quality, the whole observation period was divided into four fixed seasons: Spring (March, April, May), Summer (June, July, August), Autumn (September, October, November), and Winter (December, January, February). Twenty-two different physicochemical parameters like temperature, $\mathrm{pH}$, turbidity, electrical conductivity, specific density, total dissolved solids, total hardness, silica, phosphate, sulphate, nitrite, nitrate, ammonia, chloride, total alkalinity, magnesium hardness, calcium hardness, aluminum, iron, manganese, ozone and radical chloride were all examined. In this study, the use of descriptive statistical analysis is a must to determine minimum value, maximum value, mean and standard deviation as well as the $95 \%$ confidence intervals for exploring whether there is present any significant relationship among water quality parameters and between sites.

\subsection{Descriptive and Multivariate Statistical Methods}

Data results of water samples presented in terms of descriptive analysis for evaluating the seasonal trends between months and some environmetric techniques such as ANOVA, MANOVA and the results of multiple comparison t-test that are used to evaluate the impact of organism and their environment anthropogenic activities on spatiotemporal variations of physicochemical characteristics of the lake. Three multivariate methods were applied to spatiotemporal water quality assessment of the lake. First, Wilks' Lambda Statistics in the MANOVA techniques which have been widely used as unbiased methods in analysis of water quality data and for evaluating sites and seasons which have mean effect factor on the physiochemical of water. Second, ANOVA $(p<0.05)$ level of significance was used also to analyzing and evaluating significant differences among the sites for all variables of water quality. Third, the results of multiple comparison t-test techniques was used to find out if there are differences among the mean of physiochemical characteristics of water data due to sites and seasonal variation that drawing out meaningful conclusions. ANOVA technique explore sets of variables with similar properties and successfully allowing to simplify description of data observations and to enable to find out the structure or patterns in the presence of confusing data. SPSS (version. 22) software was used to perform for a statistical analyses and their applications. Simply, to explore the differences between the means of different sites and for describing the temporal variations of the observed water quality parameters. Also, specific various physicochemical parameters carried out in order to recognize the nature and magnitude of the relationship among them [12-13].

\section{Results and Discussion}

Physicochemical analysis and their original data measurements in seasons and sites are summarized in Table 1. Sites 1 and 4 has a higher and lower temperature in winter seasons respectively, whereas in spring seasons site 3 and 1. In summer sites 3 and 4 indicated the higher and lower values while sites 1 and 2 showed the higher and lower values. pH parameters showed in winter a higher and lower values at sites 3 and 2 while sites 3 and 5 showed the higher and lower values respectively. Sites 2 and 5 the higher value and lower values in Autumn, also sites 4 and 2 as well. Turbidities in winter appear very high at site 1 and very in site 2 in contrast in spring season sites 2 seem to be very low and the highest value appeared in site 4 . In summer and autumn seasons 
value appeared very high in sites 4 and 2 whereas the lowest have been seen in sites 5 and 5 respectively. Electrical conductivity has showed higher values at sites 1 and lower at site 4 in winter, but sites 3 showed a higher value while site 4 showed a lower value. Summer the higher value shown at site 1 but the lower value at site 3. In autumn site 1 has shown higher value while site 2 has the lowest value. Physical specific density parameter has shown the higher values in winter, spring, summer and autumn at two sites 2 and 3 , in addition to 3, 3 and two sites 1 and 3, while the lowest values have been seen at sites 5, 5, 2, 5 respectively. Finally, the total dissolved solids have shown the higher values in winter, spring, summer and autumn at sites 5, 1, 5 and 3, while the lowest values have been seen at sites 1, 3, 4 and 1 respectively.

Table 1: Physicochemical characteristics of water of Dumate Al-Jundal lake (February 2009 to January 2011)

\begin{tabular}{|c|c|c|c|c|c|c|c|}
\hline $\begin{array}{l}\text { S. } \\
\text { no. }\end{array}$ & Parameters & Seasons & Site 1 & Site 2 & Site 3 & Site 4 & Site 5 \\
\hline \multirow[t]{5}{*}{1} & \multicolumn{7}{|l|}{ Temperature ${ }^{\circ} \mathrm{C}$} \\
\hline & $\begin{array}{l}\text { winter (December, } \\
\text { January, and February) }\end{array}$ & Winter & 16.10 & 16.50 & 15.70 & 15.20 & 15.70 \\
\hline & $\begin{array}{l}\text { spring (March, April, } \\
\text { and May) }\end{array}$ & Spring & 19.50 & 21.60 & 22.20 & 19.90 & 21.60 \\
\hline & $\begin{array}{l}\text { summer (June, July, } \\
\text { and August), }\end{array}$ & Summer & 23.30 & 23.70 & 24.60 & 23.80 & 22.90 \\
\hline & $\begin{array}{l}\text { autumn } \quad \text { (September, } \\
\text { October, } \\
\text { November), and }\end{array}$ & Autumn & 22.50 & 18.70 & 21.50 & 19.60 & 21.40 \\
\hline \multirow[t]{5}{*}{2} & \multicolumn{7}{|l|}{$\mathrm{pH}$} \\
\hline & $\begin{array}{l}\text { winter (December, } \\
\text { January, and February) }\end{array}$ & Winter & 8.21 & 8.11 & 8.45 & 8.29 & 8.13 \\
\hline & $\begin{array}{l}\text { spring (March, April, } \\
\text { and May) }\end{array}$ & Spring & 8.15 & 8.34 & 8.44 & 8.19 & 8.10 \\
\hline & $\begin{array}{l}\text { summer (June, July, } \\
\text { and August), }\end{array}$ & Summer & 8.11 & 8.30 & 8.25 & 8.17 & 8.09 \\
\hline & $\begin{array}{lr}\text { autumn } & \text { (September, } \\
\text { October, } & \text { and } \\
\text { November), } & \end{array}$ & Autumn & 8.04 & 8.22 & 8.09 & 8.15 & 8.01 \\
\hline \multirow[t]{5}{*}{3} & \multicolumn{7}{|l|}{ Turbidity (NTU) } \\
\hline & $\begin{array}{l}\text { winter (December, } \\
\text { January, and February) }\end{array}$ & Winter & 2.92 & 2.09 & 2.61 & 2.57 & 2.11 \\
\hline & $\begin{array}{l}\text { spring (March, April, } \\
\text { and May) }\end{array}$ & Spring & 2.71 & 1.93 & 2.15 & 2.87 & 2.55 \\
\hline & $\begin{array}{l}\text { summer (June, July, } \\
\text { and August), }\end{array}$ & Summer & 2.45 & 2.42 & 2.38 & 2.60 & 2.28 \\
\hline & $\begin{array}{lr}\text { autumn } & \text { (September, } \\
\text { October, } & \text { and } \\
\text { November), }\end{array}$ & Autumn & 2.79 & 5.22 & 2.93 & 2.81 & 2.77 \\
\hline
\end{tabular}




\begin{tabular}{|c|c|c|c|c|c|c|c|}
\hline \multirow[t]{5}{*}{4} & $\begin{array}{l}\text { Electrical conductivity } \\
\qquad(\mu \mathrm{S} / \mathrm{cm})\end{array}$ & & & & & & \\
\hline & $\begin{array}{l}\text { winter (December, } \\
\text { January, and February) }\end{array}$ & Winter & 48549 & 48237 & 48390 & 48227 & 48387 \\
\hline & $\begin{array}{l}\text { spring (March, April, } \\
\text { and May) }\end{array}$ & Spring & 48530 & 47090 & 45990 & 45290 & 45984 \\
\hline & $\begin{array}{l}\text { summer (June, July, } \\
\text { and August), }\end{array}$ & Summer & 48640 & 48410 & 48172 & 49272 & 48262 \\
\hline & $\begin{array}{l}\text { autumn } \quad \text { (September, } \\
\text { October, } \\
\text { November), }\end{array}$ & Autumn & 49610 & 48780 & 48907 & 48997 & 48837 \\
\hline \multirow[t]{5}{*}{5} & Specific density & & & & & & \\
\hline & $\begin{array}{l}\text { winter (December, } \\
\text { January, and February) }\end{array}$ & Winter & 1.033 & 1.034 & 1.034 & 1.032 & 1.031 \\
\hline & $\begin{array}{l}\text { spring (March, April, } \\
\text { and May) }\end{array}$ & Spring & 1.032 & 1.031 & 1.033 & 1.034 & 1.030 \\
\hline & $\begin{array}{l}\text { summer (June, July, } \\
\text { and August), }\end{array}$ & Summer & 1.034 & 1.032 & 1.034 & 1.033 & 1.033 \\
\hline & $\begin{array}{l}\text { autumn (September, } \\
\text { October, } \\
\text { November), }\end{array}$ & Autumn & 1.035 & 1.035 & 1.035 & 1.034 & 1.033 \\
\hline \multirow[t]{5}{*}{6} & $\begin{array}{l}\text { Total dissolved solids } \\
\text { (mg/L) }\end{array}$ & & & & & & \\
\hline & $\begin{array}{l}\text { winter (December, } \\
\text { January, and February) }\end{array}$ & Winter & 49532 & 52620 & 49550 & 49562 & 52670 \\
\hline & $\begin{array}{l}\text { spring (March, April, } \\
\text { and May) }\end{array}$ & Spring & 52440 & 50670 & 48740 & 50670 & 48780 \\
\hline & $\begin{array}{l}\text { summer (June, July, } \\
\text { and August), }\end{array}$ & Summer & 52440 & 52050 & 51920 & 49532 & 52620 \\
\hline & $\begin{array}{l}\text { autumn } \quad \text { (September, } \\
\text { October, } \\
\text { November), }\end{array}$ & Autumn & 45090 & 49550 & 50739 & 49540 & 50639 \\
\hline \multirow[t]{5}{*}{7} & Silica $\left(\mathrm{SiO}_{4}^{-}\right) \quad(\mathrm{mg} / \mathrm{L})$ & & & & & & \\
\hline & $\begin{array}{l}\text { winter (December, } \\
\text { January, and February) }\end{array}$ & Winter & 8.40 & 6.60 & 7.30 & 7.40 & 7.50 \\
\hline & $\begin{array}{l}\text { spring (March, April, } \\
\text { and May) }\end{array}$ & Spring & 8.10 & 11.6 & 8.30 & 8.20 & 8.15 \\
\hline & $\begin{array}{l}\text { summer (June, July, } \\
\text { and August), }\end{array}$ & Summer & 11.3 & 5.20 & 5.10 & 5.12 & 5.90 \\
\hline & $\begin{array}{l}\text { autumn } \quad \text { (September, } \\
\text { October, } \\
\text { November), }\end{array}$ & Autumn & 8.20 & 7.30 & 7.50 & 7.40 & 7.48 \\
\hline
\end{tabular}




\begin{tabular}{|c|c|c|c|c|c|c|c|}
\hline \multirow[t]{5}{*}{8} & $\begin{array}{c}\text { Phosphate }\left(\mathrm{PO}_{4}^{-}\right) \\
(\mathrm{mg} / \mathrm{L})\end{array}$ & \multirow[b]{2}{*}{ Winter } & \multirow[b]{2}{*}{0.75} & \multirow[b]{2}{*}{0.57} & \multirow[b]{2}{*}{0.64} & \multirow[b]{2}{*}{0.65} & \multirow[b]{2}{*}{0.61} \\
\hline & $\begin{array}{l}\text { winter (December, } \\
\text { January, and February) }\end{array}$ & & & & & & \\
\hline & $\begin{array}{l}\text { spring (March, April, } \\
\text { and May) }\end{array}$ & Spring & 0.80 & 0.66 & 0.66 & 0.65 & 0.64 \\
\hline & $\begin{array}{l}\text { summer (June, July, } \\
\text { and August), }\end{array}$ & Summer & 0.42 & 0.55 & 0.67 & 0.67 & 0.63 \\
\hline & $\begin{array}{l}\text { autumn } \quad \text { (September, } \\
\text { October, } \\
\text { November), and }\end{array}$ & Autumn & 0.67 & 0.73 & 0.49 & 0.51 & 0.48 \\
\hline \multirow[t]{5}{*}{9} & Sulphate $\left(\mathrm{SO}_{4}^{-}\right)(\mathrm{mg} / \mathrm{L})$ & & & & & & \\
\hline & $\begin{array}{l}\text { winter (December, } \\
\text { January, and February) }\end{array}$ & Winter & 1600 & 1640 & 1767 & 1757 & 1769 \\
\hline & $\begin{array}{l}\text { spring (March, April, } \\
\text { and May) }\end{array}$ & Spring & 1903 & 2245 & 1540 & 1520 & 1530 \\
\hline & $\begin{array}{l}\text { summer (June, July, } \\
\text { and August), }\end{array}$ & Summer & 960 & 810 & 990 & 993 & 986 \\
\hline & $\begin{array}{lr}\text { autumn } & \text { (September, } \\
\text { October, } & \text { and } \\
\text { November), } & \end{array}$ & Autumn & 1552 & 1109 & 930 & 986 & 979 \\
\hline \multirow[t]{5}{*}{10} & Nitrite $\left(\mathrm{NO}_{2}^{-}\right)(\mathrm{mg} / \mathrm{L})$ & & & & & & \\
\hline & $\begin{array}{l}\text { winter (December, } \\
\text { January, and February) }\end{array}$ & Winter & 0.048 & 0.018 & 0.021 & 0.019 & 0.020 \\
\hline & $\begin{array}{l}\text { spring (March, April, } \\
\text { and May) }\end{array}$ & Spring & 0.015 & 0.014 & 0.011 & 0.013 & 0.012 \\
\hline & $\begin{array}{l}\text { summer (June, July, } \\
\text { and August), }\end{array}$ & Summer & 0.017 & 0.020 & 0.018 & 0.017 & 0.014 \\
\hline & $\begin{array}{l}\text { autumn } \quad \text { (September, } \\
\text { October, } \\
\text { November), }\end{array}$ & Autumn & 0.050 & 0.072 & 0.061 & 0.055 & 0.064 \\
\hline \multirow[t]{5}{*}{11} & Nitrate $\left(\mathrm{NO}_{3}^{-}\right)(\mathrm{mg} / \mathrm{L})$ & & & & & & \\
\hline & $\begin{array}{l}\text { winter (December, } \\
\text { January, and February) }\end{array}$ & Winter & 2.1 & 1.3 & 1.1 & 1.0 & 1.1 \\
\hline & $\begin{array}{l}\text { spring (March, April, } \\
\text { and May) }\end{array}$ & Spring & 1.6 & 1.4 & 0.9 & 1.01 & 1.2 \\
\hline & $\begin{array}{l}\text { summer (June, July, } \\
\text { and August), }\end{array}$ & Summer & 1.1 & 1.3 & 0.7 & 1.1 & 0.90 \\
\hline & $\begin{array}{l}\text { autumn } \\
\text { October, } \\
\text { November), }\end{array}$ & Autumn & 1.1 & 1.3 & 0.9 & 1.2 & 1.1 \\
\hline 12 & Ammonia $\left(\mathrm{NH}_{3}\right)(\mathrm{mg} / \mathrm{L})$ & & & & & & \\
\hline
\end{tabular}




\begin{tabular}{|c|c|c|c|c|c|c|c|}
\hline \multirow[t]{4}{*}{ 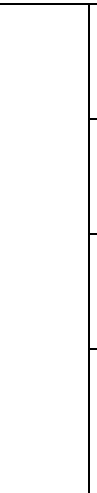 } & $\begin{array}{l}\text { winter (December, } \\
\text { January, and February) }\end{array}$ & Winter & 0.09 & 0.05 & 0.08 & 0.07 & 0.08 \\
\hline & $\begin{array}{l}\text { spring (March, April, } \\
\text { and May) }\end{array}$ & Spring & 0.04 & 0.11 & 0.08 & 0.06 & 0.07 \\
\hline & $\begin{array}{l}\text { summer (June, July, } \\
\text { and August), }\end{array}$ & Summer & 0.09 & 0.13 & 0.25 & 0.09 & 0.18 \\
\hline & $\begin{array}{l}\text { autumn } \quad \text { (September, } \\
\text { October, } \\
\text { November), }\end{array}$ & Autumn & 0.15 & 0.07 & 0.05 & 0.06 & 0.12 \\
\hline \multirow[t]{5}{*}{13} & Chloride (mg/L) & & & & & & \\
\hline & $\begin{array}{l}\text { winter (December, } \\
\text { January, and February) }\end{array}$ & Winter & 15476 & 11930 & 12920 & 11730 & 12320 \\
\hline & $\begin{array}{l}\text { spring (March, April, } \\
\text { and May) }\end{array}$ & Spring & 14600 & 16500 & 15350 & 15251 & 14358 \\
\hline & $\begin{array}{l}\text { summer (June, July, } \\
\text { and August), }\end{array}$ & Summer & 14900 & 35400 & 32300 & 35411 & 32322 \\
\hline & $\begin{array}{l}\text { autumn } \quad \text { (September, } \\
\text { October, } \\
\text { November), }\end{array}$ & Autumn & 16100 & 17800 & 13800 & 17848 & 13870 \\
\hline \multirow[t]{5}{*}{14} & $\begin{array}{c}\text { Total hardness }\left(\mathrm{CaCO}_{3}\right. \\
\mathrm{mg} / \mathrm{l})\end{array}$ & & & & & & \\
\hline & $\begin{array}{l}\text { winter (December, } \\
\text { January, and February) }\end{array}$ & Winter & 15476 & 6360 & 5340 & 5360 & 6340 \\
\hline & $\begin{array}{l}\text { spring (March, April, } \\
\text { and May) }\end{array}$ & Spring & 4683 & 2660 & 8050 & 5660 & 7050 \\
\hline & $\begin{array}{l}\text { summer (June, July, } \\
\text { and August), }\end{array}$ & Summer & 7850 & 4730 & 8026 & 7730 & 7826 \\
\hline & $\begin{array}{l}\text { autumn (September, } \\
\text { October, } \\
\text { November), }\end{array}$ & Autumn & 16100 & 17800 & 13800 & 14730 & 14026 \\
\hline \multirow[t]{5}{*}{15} & $\begin{array}{l}\text { Total alkalinity } \\
\left(\mathrm{CaCO}_{3} / \mathrm{L}\right)\end{array}$ & & & & & & \\
\hline & $\begin{array}{l}\text { winter (December, } \\
\text { January, and February) }\end{array}$ & Winter & 450 & 295 & 315 & 301 & 319 \\
\hline & $\begin{array}{l}\text { spring (March, April, } \\
\text { and May) }\end{array}$ & Spring & 320 & 340 & 600 & 349 & 330 \\
\hline & $\begin{array}{l}\text { summer (June, July, } \\
\text { and August), }\end{array}$ & Summer & 650 & 710 & 702 & 700 & 705 \\
\hline & $\begin{array}{l}\text { autumn } \quad \text { (September, } \\
\text { October, } \\
\text { November), }\end{array}$ & Autumn & 750 & 500 & 800 & 550 & 504 \\
\hline
\end{tabular}




\begin{tabular}{|c|c|c|c|c|c|c|c|}
\hline \multirow[t]{5}{*}{16} & $\begin{array}{c}\text { Magnesium Hardness } \\
(\mathrm{mg} / \mathrm{L})\end{array}$ & & & & & & \\
\hline & $\begin{array}{l}\text { winter (December, } \\
\text { January, and February) }\end{array}$ & Winter & 5838 & 1405 & 1584 & 1838 & 1455 \\
\hline & $\begin{array}{l}\text { spring (March, April, } \\
\text { and May) }\end{array}$ & Spring & 2291 & 5420 & 6320 & 5920 & 6358 \\
\hline & $\begin{array}{l}\text { summer (June, July, } \\
\text { and August), }\end{array}$ & Summer & 8300 & 7452 & 6830 & 7487 & 6839 \\
\hline & $\begin{array}{l}\text { autumn } \quad \text { (September, } \\
\text { October, } \\
\text { November), }\end{array}$ & Autumn & 5630 & 7840 & 7340 & 7878 & 7396 \\
\hline \multirow[t]{5}{*}{17} & $\begin{array}{l}\text { Calcium Hardness } \\
\text { (mg/L) }\end{array}$ & & & & & & \\
\hline & $\begin{array}{l}\text { winter (December, } \\
\text { January, and February) }\end{array}$ & Winter & 1974 & 3205 & 4120 & 3145 & 4122 \\
\hline & $\begin{array}{l}\text { spring (March, April, } \\
\text { and May) }\end{array}$ & Spring & 4625 & 2010 & 1423 & 2000 & 1451 \\
\hline & $\begin{array}{l}\text { summer (June, July, } \\
\text { and August), }\end{array}$ & Summer & 2430 & 1390 & 1700 & 1395 & 1770 \\
\hline & $\begin{array}{l}\text { autumn (September, } \\
\text { October, } \\
\text { November), }\end{array}$ & Autumn & 1050 & 1850 & 1654 & 1750 & 1647 \\
\hline \multirow[t]{5}{*}{18} & Aluminum (Al) $(\mu \mathrm{gm} / \mathrm{L})$ & & & & & & \\
\hline & $\begin{array}{l}\text { winter (December, } \\
\text { January, and February) }\end{array}$ & Winter & 0.012 & 0.009 & 0.013 & 0.009 & 0.013 \\
\hline & $\begin{array}{l}\text { spring (March, April, } \\
\text { and May) }\end{array}$ & Spring & 0.039 & 0.011 & 0.012 & 0.011 & 0.012 \\
\hline & $\begin{array}{l}\text { summer (June, July, } \\
\text { and August), }\end{array}$ & Summer & 0.007 & 0.009 & 0.016 & 0.009 & 0.016 \\
\hline & $\begin{array}{l}\text { autumn (September, } \\
\text { October, } \\
\text { November), }\end{array}$ & Autumn & 0.008 & 0.011 & 0.015 & 0.011 & 0.015 \\
\hline \multirow[t]{5}{*}{19} & Iron $(\mathrm{Fe})(\mu \mathrm{gm} / \mathrm{L})$ & & & & & & \\
\hline & $\begin{array}{l}\text { winter (December, } \\
\text { January, and February) }\end{array}$ & Winter & 0.14 & 0.04 & 0.03 & 0.04 & 0.03 \\
\hline & $\begin{array}{l}\text { spring (March, April, } \\
\text { and May) }\end{array}$ & Spring & 0.05 & 0.10 & 0.06 & 0.10 & 0.06 \\
\hline & $\begin{array}{l}\text { summer (June, July, } \\
\text { and August), }\end{array}$ & Summer & 0.07 & 0.07 & 0.11 & 0.07 & 0.11 \\
\hline & $\begin{array}{l}\text { autumn } \quad \text { (September, } \\
\text { October, } \\
\text { November), }\end{array}$ & Autumn & 0.05 & 0.11 & 0.12 & 0.11 & 0.12 \\
\hline
\end{tabular}




\begin{tabular}{|c|c|c|c|c|c|c|c|}
\hline \multirow[t]{5}{*}{20} & $\begin{array}{l}\text { Manganese (Mn) } \\
\quad(\mu \mathrm{gm} / \mathrm{L})\end{array}$ & \multirow[b]{2}{*}{ Winter } & \multirow[b]{2}{*}{0.003} & \multirow[b]{2}{*}{0.008} & \multirow[b]{2}{*}{0.003} & \multirow[b]{2}{*}{0.008} & \multirow[b]{2}{*}{0.003} \\
\hline & $\begin{array}{l}\text { winter (December, } \\
\text { January, and February) }\end{array}$ & & & & & & \\
\hline & $\begin{array}{l}\text { spring (March, April, } \\
\text { and May) }\end{array}$ & Spring & 0.01 & 0.007 & 0.002 & 0.007 & 0.002 \\
\hline & $\begin{array}{l}\text { summer (June, July, } \\
\text { and August), }\end{array}$ & Summer & 0.003 & 0.002 & 0.003 & 0.002 & 0.003 \\
\hline & $\begin{array}{l}\text { autumn } \quad \text { (September, } \\
\text { October, } \\
\text { November), and }\end{array}$ & Autumn & 0.002 & 0.001 & 0.002 & 0.001 & 0.002 \\
\hline \multirow[t]{5}{*}{21} & Ozone $\left(\mathrm{O}_{3}\right)(\mu \mathrm{gm} / \mathrm{L})$ & & & & & & \\
\hline & $\begin{array}{l}\text { winter (December, } \\
\text { January, and February) }\end{array}$ & Winter & 0.0 & 0.0 & 0.0 & 0.0 & 0.0 \\
\hline & $\begin{array}{l}\text { spring (March, April, } \\
\text { and May) }\end{array}$ & Spring & 0.0 & 0.0 & 0.0 & 0.0 & 0.0 \\
\hline & $\begin{array}{l}\text { summer (June, July, } \\
\text { and August), }\end{array}$ & Summer & 0.0 & 0.0 & 0.0 & 0.0 & 0.0 \\
\hline & $\begin{array}{lr}\text { autumn } & \text { (September, } \\
\text { October, } & \text { and } \\
\text { November), }\end{array}$ & Autumn & 0.0 & 0.0 & 1.20 & 0.0 & 0.0 \\
\hline \multirow[t]{5}{*}{22} & $\mathrm{Cl}(\mu \mathrm{gm} / \mathrm{L})$ radical & & & & & & \\
\hline & $\begin{array}{l}\text { winter (December, } \\
\text { January, and February) }\end{array}$ & Winter & 0.0 & 0.0 & 0.0 & 0.0 & 0.0 \\
\hline & $\begin{array}{l}\text { spring (March, April, } \\
\text { and May) }\end{array}$ & Spring & 0.0 & 0.0 & 0.0 & 0.0 & 0.0 \\
\hline & $\begin{array}{l}\text { summer (June, July, } \\
\text { and August), }\end{array}$ & Summer & 0.0 & 0.0 & 0.0 & 0.0 & 0.0 \\
\hline & $\begin{array}{lr}\text { autumn } & \text { (September, } \\
\text { October, } & \text { and } \\
\text { November), } & \end{array}$ & Autumn & 0.0 & 0.0 & 0.0 & 0.0 & 0.0 \\
\hline
\end{tabular}

The chemical parameters like silica indicated the higher values in winter, spring, summer and autumn at sites 1 , 2, 1 and 1, while the lowest values have been seen at sites 2, 1,2 and 2 respectively. Also, phosphate indicated the higher values in winter, spring, summer and autumn at sites 1, 1, 3 and 4 and 2, while the lowest values have been seen at sites 5, 5, 1 and 5 respectively. Sulphate indicated the higher values in winter, spring, summer and autumn at sites 4, 2, 4 and 1, while the lowest values have been seen at sites 1, 4, 2 and 3 respectively. Nitrite indicated the higher values in winter, spring, summer and autumn at sites 5, 1, 3 and 2, while the lowest values have been seen at sites 2, 3, 1 and 4 and 1 respectively. Nitrate indicated the higher values in winter, spring, summer and autumn at sites 1, 1,2 and 2, while the lowest values have been seen at sites 4, 3, 3 and 3 respectively. Ammonia indicated the higher values in winter, spring, summer and autumn at sites 1, 2, 3 and 1, while the lowest values have been seen at sites 2, 1, 1 and 4 and 3 respectively. Chloride indicated the higher values in winter, spring, summer and autumn at sites 1, 3, 4 and 4, while the lowest values have been seen at sites 4, 5, 1 and 5 respectively. Total hardness indicated the higher values in winter, spring, summer and autumn 
at sites 1, 3, 3 and 2, while the lowest values have been seen at sites 3,2, 2 and 3 respectively. Total alkalinity indicated the higher values in winter, spring, summer and autumn at sites 1, 3,2 and 1 while the lowest values have been seen at sites 2, 1, 1 and 2, respectively. Magnesium Hardness indicated the higher values in winter spring, summer and autumn at sites 1, 5, 1 and 4 while the lowest values have been seen at sites 2, 1, 3 and 1 respectively. Calcium hardness indicated the higher values in winter, spring, summer and autumn at sites 5, 1, 1 and 2 while the lowest values have been seen at sites 1, 3, 2 and 1, respectively. Aluminum indicated the higher values in winter, spring, summer and autumn at sites 5, 1, 3 and 5 and 3 and 5 while the lowest values have been seen at sites 2 and 4, 2, 4 and 2 and 4 respectively. Iron indicated the higher values in winter, spring, summer and autumn at sites 1,3,5, 1 and 3, 5 while the lowest values have been seen at sites 3, 5, 1, 2, 4 and 1 respectively. Manganese indicated the higher values in winter, spring, summer and autumn at sites $1,3,5,2,4$, 2,4 and 2 while the lowest values have been seen at sites $2,4,1,2,4,2,4$ and 2 respectively. While ozone and chloride radical showed zero values.

\subsection{Physical parameters}

The descriptive analysis data of the physical parameters, Table 2 and Fig. 3, 4 shows, maximum and minimum temperature values were recorded $24.6-15.2{ }^{\circ} \mathrm{C}$ with mean $20.30{ }^{\circ} \mathrm{C}$ respectively. The warmest temperature was noticed at August in summer and the coldest at January in winter seasons. Buffering capacity $(\mathrm{pH})$ of a lake that measure its relative acidity or alkalinity exhibit wide variations, not only in actual $\mathrm{pH}$ values, but also in the amount of dissolved materials that impact $\mathrm{pH}$ which was ranged from 8.010 to 8.450 with the highest values in spring season and the lowest in autumn season at most of the study sites. For instance, the pH values at site 5 of the lake showed a trend decreasing from wet to dry season, while it becomes higher during the dry season. The reason behind extensive variations was due to the limestone, which contributes calcium carbonate to the water of the lake, so the mean of total hardness $(\mathrm{CaCO} 3)$ is about $6860.023 \mathrm{mg} / \mathrm{L}$ responsible for the excellent buffering capacity of the lake. 
Table 2: Statistical measurements for the physiochemical parameters data of water samples.

\begin{tabular}{|c|c|c|c|c|c|c|c|}
\hline \multirow[b]{2}{*}{ Parameter } & \multirow{2}{*}{$\mathrm{N}$} & \multirow[b]{2}{*}{ Minimum } & \multirow[b]{2}{*}{ Maximum } & \multirow[b]{2}{*}{ Mean } & \multirow[b]{2}{*}{ Std. Deviation } & \multicolumn{2}{|c|}{$\begin{array}{l}\text { 95\% Confidence Interval for } \\
\text { Mean }\end{array}$} \\
\hline & & & & & & Lower Bound & $\begin{array}{l}\text { Upper } \\
\text { Bound }\end{array}$ \\
\hline Temperature ${ }^{\circ} \mathrm{C}$ & 20 & 15.200 & 24.600 & 20.30000 & 3.050453 & 18.87234 & 21.72766 \\
\hline $\mathrm{pH}$ & 20 & 8.010 & 8.450 & 8.19200 & .121940 & 8.13493 & 8.24907 \\
\hline $\begin{array}{l}\text { Turbidity (NTU) Nephlometric } \\
\text { Turbidity Units }\end{array}$ & 20 & 1.930 & 5.220 & 2.65800 & .671343 & 2.34380 & 2.97220 \\
\hline Electrical conductivity $(\mu \mathrm{S} / \mathrm{cm})$ & 20 & 45290.000 & 49610.000 & 48128.05000 & 1145.979860 & 47591.71492 & $\begin{array}{c}48664.3850 \\
8\end{array}$ \\
\hline Specific density (mg/ml) & 20 & 1.030 & 1.035 & 1.03310 & .001410 & 1.03244 & 1.03376 \\
\hline Total dissolved solids (mg/L) & 20 & 45090.000 & 52670.000 & 50467.70000 & 1871.697347 & 49591.71868 & $\begin{array}{c}51343.6813 \\
2\end{array}$ \\
\hline Silica $\left(\mathrm{SiO}_{4}^{-}\right) \quad(\mathrm{mg} / \mathrm{L})$ & 20 & 5.100 & 11.600 & 7.60250 & 1.692665 & 6.81031 & 8.39469 \\
\hline Phosphate $\left(\mathrm{PO}_{4}^{-}\right)(\mathrm{mg} / \mathrm{L})$ & 20 & .420 & .800 & .62250 & .094806 & .57813 & .66687 \\
\hline Sulphate $\left(\mathrm{SO}_{4}^{-}\right)(\mathrm{mg} / \mathrm{L})$ & 20 & 810.000 & 2245.000 & 1378.30000 & 412.220061 & 1185.37507 & 1571.22493 \\
\hline
\end{tabular}




\begin{tabular}{|c|c|c|c|c|c|c|c|}
\hline Nitrite $\left(\mathrm{NO}_{2}^{-}\right) \quad(\mathrm{mg} / \mathrm{L})$ & 20 & .011 & .072 & .02895 & .020451 & .01938 & .03852 \\
\hline Nitrate $\left(\mathrm{NO}_{3}{ }^{-}\right)(\mathrm{mg} / \mathrm{L})$ & 20 & .700 & 2.100 & 1.17050 & .297241 & 1.03139 & 1.30961 \\
\hline Ammonia $\left(\mathrm{NH}_{3}\right)(\mathrm{mg} / \mathrm{L})$ & 20 & .040 & .250 & .09600 & .050513 & .07236 & .11964 \\
\hline Chloride (mg/L) & 20 & 11730.000 & 35411.000 & 18509.30000 & 8080.371670 & 14727.56965 & $\begin{array}{c}22291.0303 \\
5\end{array}$ \\
\hline Total hardness $\left(\mathrm{CaCO}_{3}\right)(\mathrm{mg} / \mathrm{L})$ & 20 & 2660.000 & 17800.000 & 8979.85000 & 4529.391574 & 6860.02949 & $\begin{array}{c}11099.6705 \\
1\end{array}$ \\
\hline Total alkalinity $\left(\mathrm{CaCO}_{3}\right)(\mathrm{mg} / \mathrm{L})$ & 20 & 295.000 & 800.000 & 509.50000 & 179.685837 & 425.40444 & 593.59556 \\
\hline Magnesium Hardness (mg/L) & 20 & 1405.000 & 8300.000 & 5571.05000 & 2417.771603 & 4439.49806 & 6702.60194 \\
\hline alcium Hardness (mg/L) & 20 & 1050.000 & 4625.000 & 2235.55000 & 1038.068524 & 1749.71898 & 2721.38102 \\
\hline Aluminum (Al) $(\mu \mathrm{gm} / \mathrm{L})$ & 20 & .007 & .039 & .01290 & .006672 & .00978 & .01602 \\
\hline Iron $(\mathrm{Fe})(\mu \mathrm{gm} / \mathrm{L})$ & 20 & .030 & .140 & .07950 & .034255 & .06347 & .09553 \\
\hline Manganese $(\mathrm{Mn})(\mu \mathrm{gm} / \mathrm{L})$ & 20 & .001 & .010 & .00370 & .002677 & .00245 & .00495 \\
\hline Ozone $\left(\mathrm{O}_{3}\right)(\mu \mathrm{gm} / \mathrm{L})$ & 20 & .000 & 1.200 & .06000 & .268328 & -.06558 & .18558 \\
\hline $\mathrm{Cl}(\mu \mathrm{gm} / \mathrm{L})$ radical & 20 & .000 & .000 & .00000 & .000000 & .00000 & .00000 \\
\hline
\end{tabular}


Generally, Table 1 shows the mean values of turbidities in the lake sites ranged from about 1.93 to 5.22 with mean 2.66 NTU (Nephlometric Turbidity Units). Whereas, the higher results appeared in October. Factors affecting like suspended matter or impurities include clay, silt, finely divided organic and inorganic matter, soluble colored organic compounds, plankton and other microscopic organisms might be led to higher turbidity from the lower and upper bounds at all sites but highest values associated with an Autumn seasonal cycle $[13,14]$. Electrical conductivity values of the lake sites Table 3, ranged from 45290.00 to 49610.00 with mean and standard deviation of 48128.05 and $1145.98 \mu \mathrm{S} / \mathrm{cm}$ were reflected in general the higher values at all the sampling sites but decreases in spring season. A progressive decrease in EC in March and spring season (see Fig. 3 and 4).

Table 3: Effects (Multivariate Test Using Wilks' Statistics) of Sites and Seasons on the physicochemical parameters.

\begin{tabular}{|c|c|c|c|}
\hline Parameter & Value & $\mathrm{F}$ & Sig. \\
\hline Temperature oC & .162 & $.606^{b}$ & .779 \\
\hline $\mathrm{pH}$ & .030 & $.591^{\mathrm{b}}$ & .824 \\
\hline Specific density & .414 & $1.443^{b}$ & 217 \\
\hline Total dissolved solids (mg/L) & .083 & $.705^{b}$ & .768 \\
\hline Silica $\left(\mathrm{SiO}_{4}^{-}\right) \quad(\mathrm{mg} / \mathrm{L})$ & .052 & $.675^{\mathrm{b}}$ & .782 \\
\hline Phosphate $\left(\mathrm{PO}_{4}^{-}\right)(\mathrm{mg} / \mathrm{L})$ & .012 & $2.379^{b}$ & .101 \\
\hline Sulphate $\left(\mathrm{SO}_{4}^{-}\right)(\mathrm{mg} / \mathrm{L})$ & .020 & $2.722^{b}$ & .448 \\
\hline Nitrite $\left(\mathrm{NO}_{2}^{-}\right) \quad(\mathrm{mg} / \mathrm{L})$ & .063 & $.598^{\mathrm{b}}$ & .837 \\
\hline Nitrate $\left(\mathrm{NO}_{3}^{-}\right)(\mathrm{mg} / \mathrm{L})$ & .338 & $.719^{\mathrm{b}}$ & .754 \\
\hline Ammonia (NH3) (mg/L) & 200 & $.787^{\mathrm{b}}$ & .701 \\
\hline Aluminum (Al) $(\mu \mathrm{gm} / \mathrm{L})$ & 187 & $1.643^{b}$ & .146 \\
\hline Iron $(\mathrm{Fe})(\mu \mathrm{gm} / \mathrm{L})$ & .058 & $3.940^{b}$ & $.002^{*}$ \\
\hline Manganese $(\mathrm{Mn})(\mu \mathrm{gm} / \mathrm{L})$ & .379 & $1.621^{b}$ & .156 \\
\hline Ozone(O3) $(\mu \mathrm{gm} / \mathrm{L})$ & .905 & $.890^{\mathrm{b}}$ & .429 \\
\hline
\end{tabular}

*Significant at the 0.05 level. 


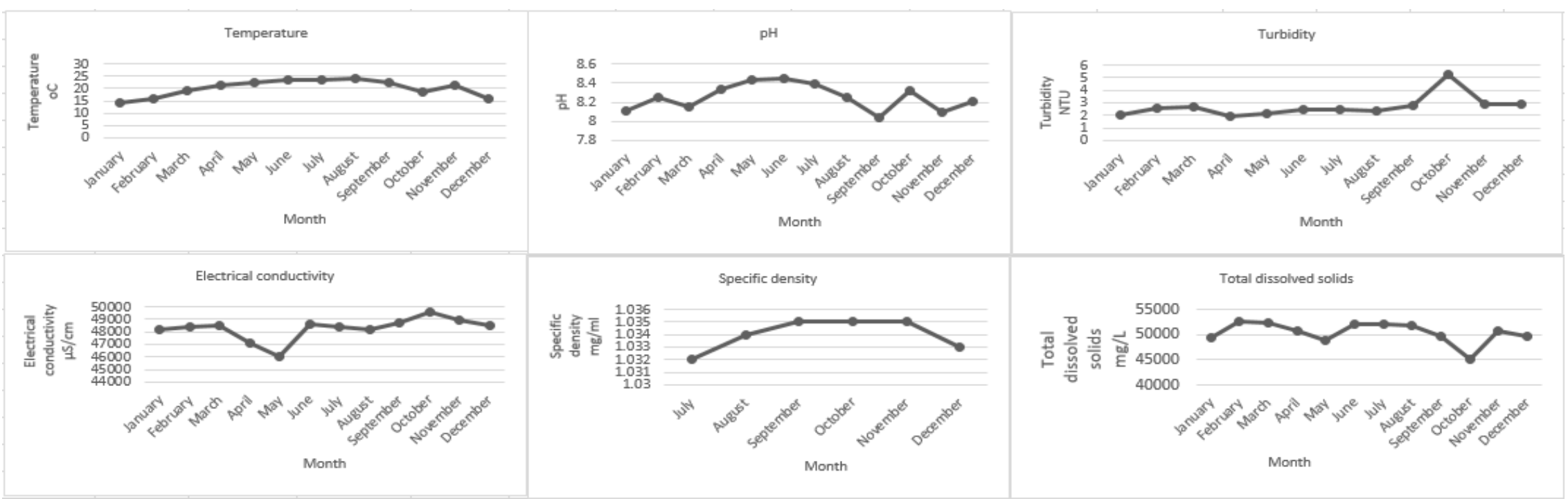

Figure 3: The mean measurements of the physical parameters according to monthly variation for all sites during February 2009 to January 2011. 


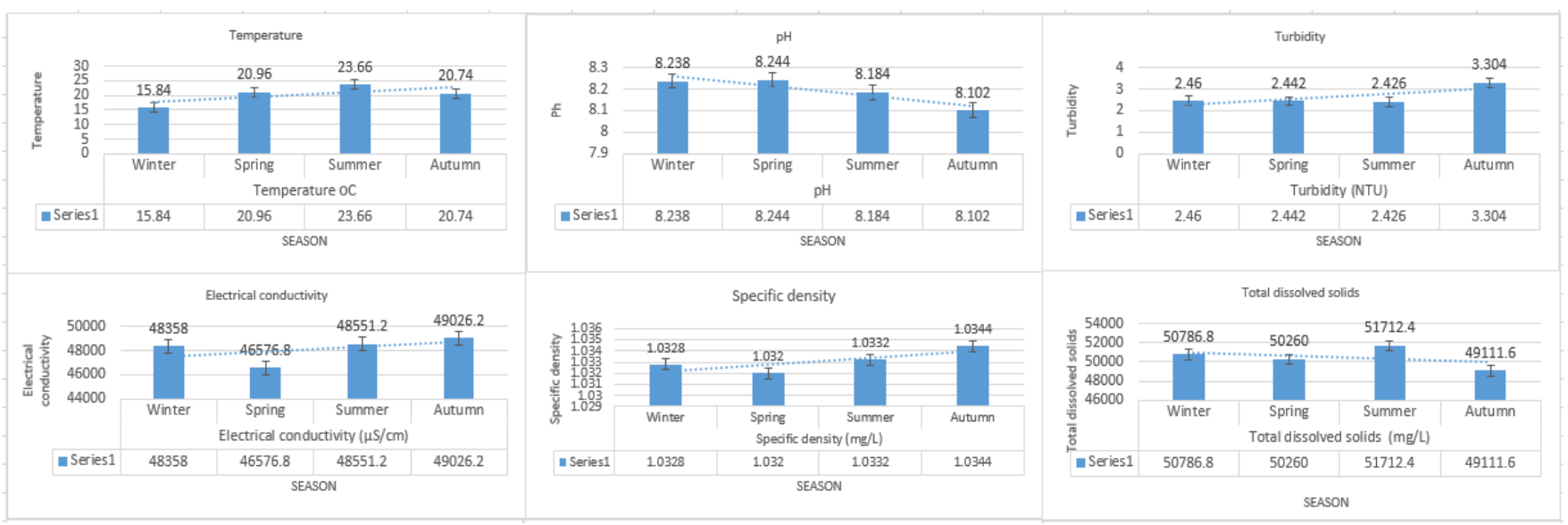

Figure 4: The mean measurements of the physical parameters according to seasonal variation for all sites during February 2009 to January 2011.

Variation in EC was significant among sites and higher values of EC were recorded in Autumn at the site 1 and lower in spring at the site 5 . The reason behinds the higher results may be attributed to seasonal driven changes that associated with highly saline water of the lake. The high salt concentration of water shows that significant dissolution and /or precipitation reactions are taking place in the lake depending upon the solubility constants of different minerals present in the lake. The standard deviation of 0.00141 and mean of 1.033 of water specific density represent a significant variation at all the sites associated with a seasonal temporally changes. Maximum water specific density for the 1,2 and 3 sites were recorded maximum of $1.035 \mathrm{gm} / \mathrm{ml}$ in autumn season and minimum in winter season (rainy season) as site $51.030 \mathrm{gm} / \mathrm{ml}$. April and September showed the lowest and the maximum values whereas autumn and spring as well. Total suspended solids of the lake water ranged between minimum 45090.0 to maximum $52670.0 \mathrm{mg} / \mathrm{l}$. The run off the agriculture wastes may be responsible for the highest values of TDS in the lake sites. Total dissolved solids show slight greater variability between seasonal cycle as indicated a lower result in spring and upper in Autumn (Fig. 3, 4).

\subsection{Chemical parameters}

Silica concentration was ranged from minimum 5.10 and maximum $11.60 \mathrm{mg} / \mathrm{l}$ with highest observation in April and lowest in July. Whereas, a lower results value in summer but higher in spring. Phosphate ion ranged from $0.42-0.80 \mathrm{mg} / \mathrm{L}$ with highest values in March and lowest in June. The highest values might be due to the existences of Organic- $\mathrm{N}$ and total nitrogen were found in summer and winter at specific sites (Table 1 and Fig. $5,6)$. On the other hand, sulphate ion was showed concentration ranged from $810.00-2245.00 \mathrm{mg} / \mathrm{l}$ in all lake sites with highest values in April and lowest in July. Spring season indicated the higher results compared with lowest in summer. Nitrite, nitrate and ammonia are all forms of

nitrogen that can be found throughout the environmental ecosystem such as soil, air, plant and water, therefore nitrogen is an essential components of plant macronutrient that exists in many forms under study [15]. Nitrite ion was showed concentration ranged from 0.011 minimum 0.072 to maximum, specifically, January represent the lowest whereas October show the highest results as well as the lowest spring and highest in Autumn seasons. While nitrate ion was concentration ranged from minimum 0.70 to maximum 2.10, August represent the lowest whereas December show the highest results as well as the lowest summer and highest in winter seasons. But the molecular ammonia showed concentration ranged from minimum 0.040 to maximum0.25mg/l. March was the lowest whereas September represented the highest results as well as the lowest in spring and highest values in summer seasons. In other words, slightly variation of nitrite specifically started from January to August then increased for September and October whereas the value decreased in November and December, the higher changes associated with spring as seen in Table 1 and Fig. 5 and 6. Chloride radical and ozone was not observed at all. 
Significantly total hardness was ranged from 17800.00 to 2660.00 according to classification based on $\mathrm{CaCO}_{3}$ $\mathrm{mg} / \mathrm{l}$. All lake sites indicated higher ascending of total hardness of water except March, April July and decreasing in September and December while Autumn has higher values compared with other seasons as seen in Table 1 and Fig. 5 and 6. This finding is in agreement with other studies for example on Washita River at Oklahoma (Madden and Morris, 1978) and disagree with the study on Moro Lake in Nigeria [14, 15]. Also, result of total alkalinities for all sites seemed to be high with higher mean and standard deviation 509.500 and $179.69 \mathrm{mg} / \mathrm{l}$ as seen in Table 1 and Fig. 5 and 6. Calcium carbonate $\left(\mathrm{CaCO}_{3}\right)$ precipitate is the major byproduct of the "whiting effect" $[16,17,18]$. The cause of the whiting effect, or whiting phenomenon, has not yet been fully explained by scientists. Some investigators believe warm temperatures initiate the production of phytoplankton, which causes a change in $\mathrm{pH}$ and results in the whiting effect. Other investigators suggest that carbonate precipitate out due to ground water flowing through carbonate containing rock limestone and discharging into surface waters, so that the results showed monthly higher ascending values except October and December whereas Spring season showed the higher values climate changes [19-20]. Concentration results of heavy metals like manganese were significantly low in all lake sites, while aluminum was totally disappeared in lake side 4 but was very low in lake sites 1,2 and 5 . Whereas results of iron concentration in all sites showed some higher ascending variations, specifically the concentration at Autumn season was slightly high compared with other seasons see Table 1 and figure 5 and $6[21,22]$.

lons in water lake like calcium and-magnesium showed significant high concentration in different lake sites and the $95 \%$ confidence interval for lower and upper bounds of mean was ranged from 1749.72 to 2721.38 and 4439.49 to $6702.60 \mathrm{mg} / \mathrm{l}$, respectively, also monthly increasing from January to June then decreasing from July to September was seen which means it was effected by the seasonal change. The major sources of these positive ions are from salts that have leached from mineral of soils and can also originate from anthropogenic sources, such as from road salt and agriculture run-off $[23,24,25]$.

The multivariate statistical methods Wilks' Lambda is a measure of the percent variance in dependent variables not explained by differences in levels of the independent variable, Table 3 summarized the data of the physiochemical parameters. Therefore, the mean effect was tested for both dependent variables of sites and the seasons using this statistics techniques in the MANOVA test which is similar to the F-test statistic in ANOVA, then can be explored if there are differences between means of group sites among seasons for a particular combination of dependent physiochemical parameters. The data observation of water sample shows both sites and seasons have mean effect only for iron ( $\mathrm{Fe} \mu \mathrm{gm} / \mathrm{L}$ ) parameter on the physiochemical of water data. Whereas, there are no effects for the other parameters. Note that some of the parameters are not appear in the table due to their small values and zero observations as seen in Table 1 for ozone and chloride radical parameters. For this reason, the use of the ANOVA procedures was required for testing each dependent variables individually at the site and the season. The test of the existence of the mean differences among the physiochemical characteristics of water data based on sites like the multiple comparisons test to test the differences between the sites for each parameter. 

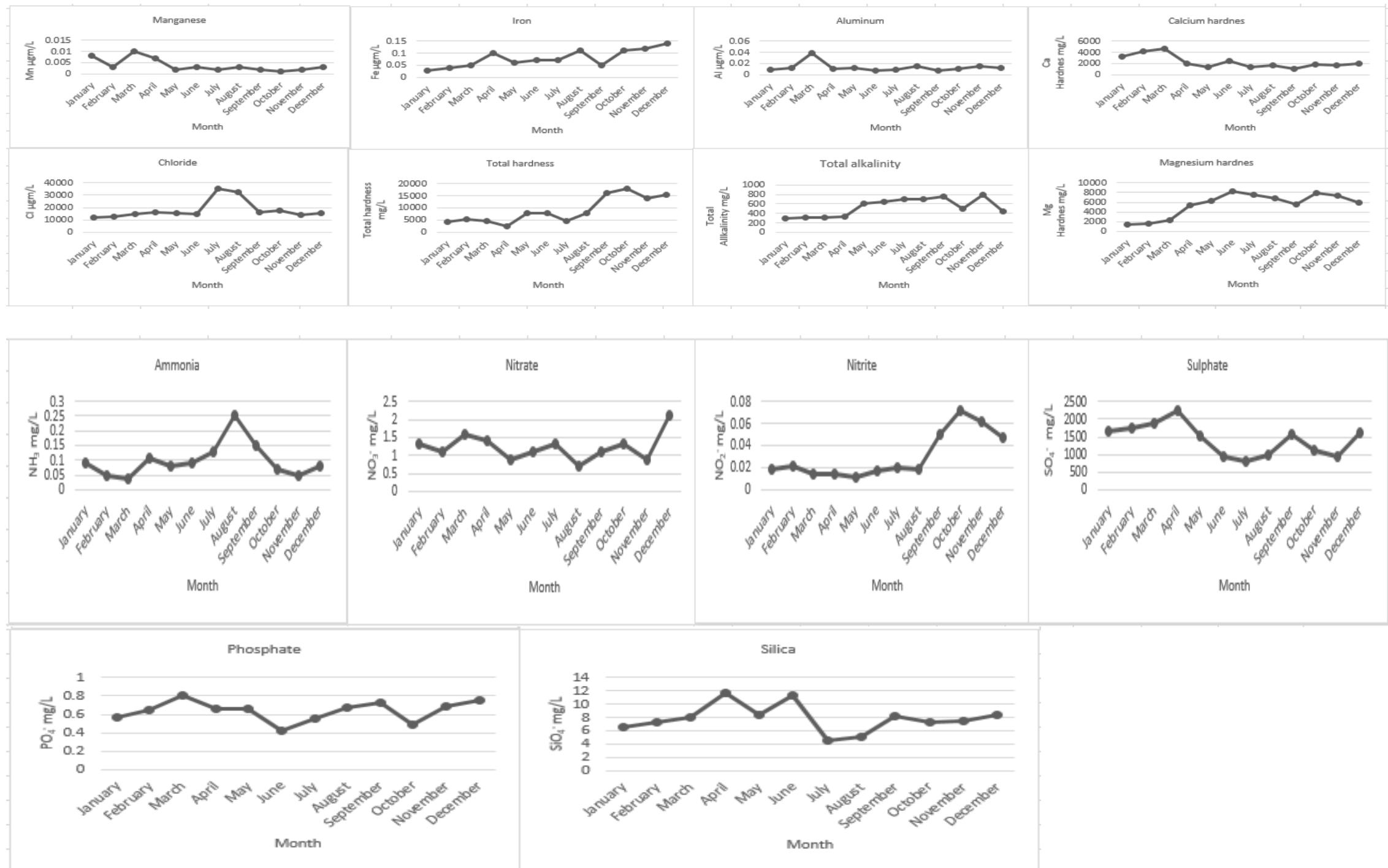

Figure 5: The mean concentrations of the chemical parameters according to monthly variation for all sites during February 2009 to January 2011. 


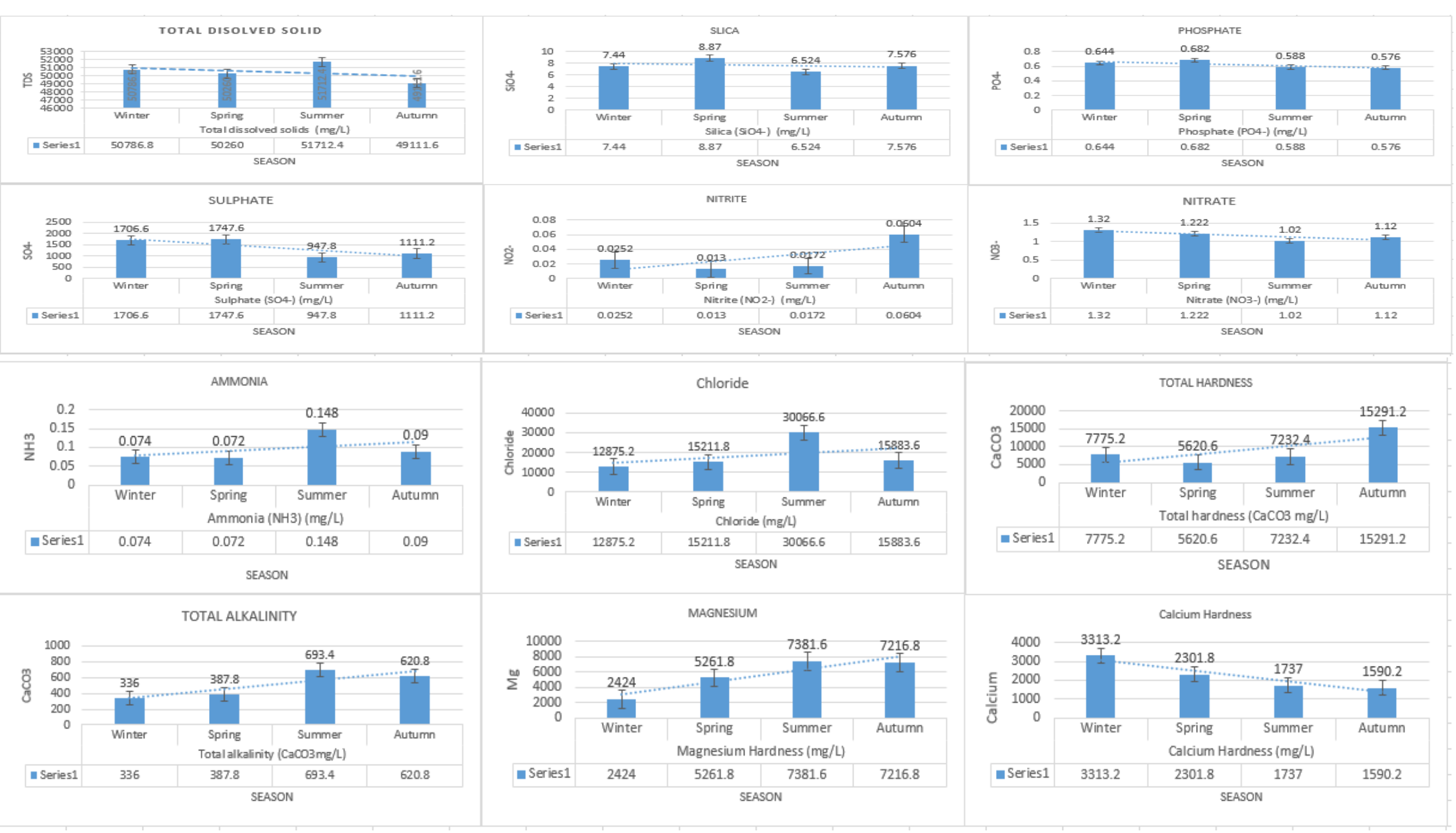

Figure 6: The mean concentrations of the chemical parameters according to seasonal variation for all sites during February 2009 to January 2011. 
Table 4: One-Way ANOVA for the mean differences of data samples according to sites for each parameter.

\begin{tabular}{|c|c|c|}
\hline Parameter & $\mathrm{F}$ & Sig. \\
\hline Temperature oC & .086 & .986 \\
\hline $\mathrm{pH}$ & 3.125 & $.047^{\star}$ \\
\hline Turbidity (NTU) & .273 & .891 \\
\hline Electrical conductivity $(\mu \mathrm{S} / \mathrm{cm})$ & .448 & .772 \\
\hline Specific density & 1.599 & .226 \\
\hline Total dissolved solids ( $\mathrm{mg} / \mathrm{l})$ & .480 & .750 \\
\hline Silica $\left(\mathrm{SiO}_{4}^{-}\right) \quad(\mathrm{mg} / \mathrm{l})$ & .933 & .471 \\
\hline Phosphate $\left(\mathrm{PO}_{4}^{-}\right)(\mathrm{mg} / \mathrm{l})$ & .238 & .912 \\
\hline Sulphate $\left(\mathrm{SO}_{4}^{-}\right)(\mathrm{mg} / \mathrm{l})$ & .166 & .953 \\
\hline Nitrite $\left(\mathrm{NO}^{-}{ }_{2}\right) \quad(\mathrm{mg} / \mathrm{l})$ & .056 & .994 \\
\hline Nitrate $\left(\mathrm{NO}_{3}^{-}\right)(\mathrm{mg} / \mathrm{l})$ & 3.668 & $.028^{*}$ \\
\hline Ammonia $\left(\mathrm{NH}_{3}\right)(\mathrm{mg} / \mathrm{l})$ & .473 & .755 \\
\hline Chloride (mg/l) & .212 & .928 \\
\hline Total hardness $\left(\mathrm{CaCO}_{3} / \mathrm{l}\right)$ & .238 & .912 \\
\hline Total alkalinity $\left(\mathrm{CaCO}_{3} / \mathrm{l}\right)$ & .425 & .788 \\
\hline Magnesium Hardness (mg/l) & .007 & 1.000 \\
\hline Calcium Hardness (mg/l) & .092 & .984 \\
\hline Aluminum (Al) $(\mu \mathrm{gm} / \mathrm{l})$ & .674 & .620 \\
\hline Iron (Fe) $(\mu \mathrm{gm} / \mathrm{l})$ & .003 & 1.000 \\
\hline Manganese $(\mathrm{Mn})(\mu \mathrm{gm} / \mathrm{l})$ & .615 & .658 \\
\hline Ozone(O3) ( $\mu \mathrm{gm} / \mathrm{l})$ & 1.000 & .438 \\
\hline $\mathrm{Cl}(\mu \mathrm{gm} / \mathrm{l})$ radical & $-\cdots-$ & $-\cdots$ \\
\hline
\end{tabular}

*Significant at the 0.05 level. 
At significant level 0.05 , Table 4 shows that there are differences among the mean of physiochemical characteristics of water data of sites only for two parameters $\mathrm{pH}$ and nitrate $\left(\mathrm{NO}_{3}^{-} \mathrm{mg} / \mathrm{L}\right)$, while there are no differences among the mean of water data analysis of sites for the other parameters under study. More specifically the results of multiple comparisons in Table 5 shows that there are slightly differences between the means of Sites 1 and 3 and Sites 2 and 5 as well as between Sites 3 and 5 for the parameter pH. In addition, there are differences between the means of Site 1 and all of Site 3, 4 and 5 as well as between Site 2 and 3 for the parameter nitrate $\left(\mathrm{NO}_{3}{ }^{-} \mathrm{mg} / \mathrm{L}\right)$. These results of analysis are shown in Table 3 and Table 4 . The multiple comparisons test was applied also to test the differences between the seasonal variation for each parameter and to evaluate the existence of the mean differences among the physiochemical characteristics of water data based on specific season.

Table 5: Multiple Comparisons Test for testing the differences between sites.

\begin{tabular}{|c|c|c|c|c|c|c|}
\hline \multirow[t]{2}{*}{ Parameter } & \multirow[t]{2}{*}{ (I) Sites } & \multirow[t]{2}{*}{ (J) Sites } & \multirow[t]{2}{*}{ Std. Error } & \multirow[t]{2}{*}{ Sig. } & \multicolumn{2}{|c|}{ 95\% Confidence Interval } \\
\hline & & & & & $\begin{array}{l}\text { Lower } \\
\text { Bound }\end{array}$ & Upper Bound \\
\hline \multirow[t]{3}{*}{$\mathrm{pH}$} & Site 1 & Site 3 & .071671 & .024 & -.33276 & -.02724 \\
\hline & Site 2 & Site 5 & .071671 & .041 & .00724 & .31276 \\
\hline & Site 3 & Site 5 & .071671 & .007 & .07224 & .37776 \\
\hline \multirow[t]{4}{*}{ Nitrate $\left(\mathrm{NO}_{3}^{-}\right)(\mathrm{mg} / \mathrm{L})$} & Site 1 & Site 3 & .168184 & .004 & .21652 & .93348 \\
\hline & & Site 4 & .168184 & .032 & .03902 & .75598 \\
\hline & & Site 5 & .168184 & .031 & .04152 & .75848 \\
\hline & Site 2 & Site 3 & .168184 & .023 & .06652 & .78348 \\
\hline
\end{tabular}

*Significant at the 0.05 level.

Table 6 shows at significant level 0.05, that there are differences among the mean of physiochemical characteristics of water data of seasons for the following parameters: temperature, electrical conductivity, specific density, sulphate $\left(\mathrm{SO}_{4}^{-} \mathrm{mg} / \mathrm{l}\right)$, nitrite $\left(\mathrm{NO}_{2}{ }^{-} \mathrm{mg} / \mathrm{l}\right)$, ammonia $\left(\mathrm{NH}_{3} \mathrm{mg} / \mathrm{l}\right)$, chloride $(\mathrm{mg} / \mathrm{l})$, total hardness $\left(\mathrm{CaCO}_{3} \mathrm{mg} / \mathrm{l}\right)$, total alkalinity $\left(\mathrm{CaCO}_{3} \mathrm{mg} / \mathrm{l}\right)$, magnesium hardness $(\mathrm{mg} / \mathrm{l})$, calcium hardness $(\mathrm{mg} / \mathrm{l})$ and manganese $(\mathrm{Mn} \mu \mathrm{gm} / \mathrm{l})$, while there are no differences among the mean of physiochemical characteristics of water data of season for the other parameters under study. In particular, the results of multiple comparisons show that there are differences between the means of Season (i) and Season (j), for each parameter described in Table 7 at significant level 0.05. These analysis of results is shown in Table 6 and Table 7.

Table 6: The One-Way ANOVA for the mean differences of data samples according to seasons for each parameter.

\begin{tabular}{|l|c|c|}
\hline \multicolumn{1}{|c|}{ Parameter } & F & Sig. \\
\hline Temperature $^{\circ} \mathrm{C}$ & 47.796 & $.000^{*}$ \\
\hline $\mathrm{pH}$ & 1.591 & .231 \\
\hline Turbidity (NTU) & 2.571 & .090 \\
\hline Electrical conductivity $(\mu \mathrm{S} / \mathrm{cm})$ & 11.889 & $.000^{*}$ \\
\hline
\end{tabular}




\begin{tabular}{|c|c|c|}
\hline Specific density & 3.509 & $.040^{*}$ \\
\hline Total dissolved solids $(\mathrm{mg} / \mathrm{l})$ & 1.927 & .166 \\
\hline Silica $\left(\mathrm{SiO}_{4}^{-}\right) \quad(\mathrm{mg} / \mathrm{l})$ & 1.844 & .180 \\
\hline Phosphate $\left(\mathrm{PO}^{-}{ }_{4}\right)(\mathrm{mg} / \mathrm{l})$ & 1.464 & .262 \\
\hline Sulphate $\left(\mathrm{SO}_{4}^{-}\right)(\mathrm{mg} / \mathrm{l})$ & 18.437 & $.000^{*}$ \\
\hline Nitrite $\left(\mathrm{NO}_{2}^{-}\right) \quad(\mathrm{mg} / \mathrm{l})$ & 38.415 & $.000^{*}$ \\
\hline Nitrate $\left(\mathrm{NO}_{3}^{-}\right)(\mathrm{mg} / \mathrm{l})$ & .938 & .445 \\
\hline Ammonia $\left(\mathrm{NH}_{3}\right)(\mathrm{mg} / \mathrm{l})$ & 3.437 & $.042^{*}$ \\
\hline Chloride (mg/l) & 15.015 & $.000^{\star}$ \\
\hline Total hardness $\left(\mathrm{CaCO}_{3} \mathrm{mg} / \mathrm{l}\right)$ & 13.281 & $.000^{\star}$ \\
\hline Total alkalinity $\left(\mathrm{CaCO}_{3} \mathrm{mg} / \mathrm{l}\right)$ & 15.393 & $.000^{*}$ \\
\hline Magnesium Hardness (mg/l) & 13.691 & $.000^{\star}$ \\
\hline Calcium Hardness (mg/l) & 4.313 & $.021^{*}$ \\
\hline Aluminum (Al) $(\mu \mathrm{gm} / \mathrm{l})$ & .829 & .497 \\
\hline Iron $(\mathrm{Fe})(\mu \mathrm{gm} / \mathrm{l})$ & 1.812 & .185 \\
\hline Manganese $(\mathrm{Mn})(\mu \mathrm{gm} / \mathrm{l})$ & 3.569 & $.038^{\star}$ \\
\hline Ozone(O3) $(\mu \mathrm{gm} / \mathrm{l})$ & 1.000 & .418 \\
\hline $\mathrm{Cl}(\mu \mathrm{gm} / \mathrm{l})$ radical & ----. & ----. \\
\hline
\end{tabular}

*Significant at the 0.05 level.

Table 7: Multiple Comparisons Test for testing the differences between seasons

\begin{tabular}{|c|c|c|c|c|c|c|c|}
\hline \multirow[t]{2}{*}{ Parameter } & \multirow[t]{2}{*}{ (I) Season } & \multirow[t]{2}{*}{ (J) Season } & \multirow[t]{2}{*}{ Mean Difference (I-J) } & \multirow[t]{2}{*}{ Std. Error } & \multirow[t]{2}{*}{ Sig. } & \multicolumn{2}{|c|}{ 95\% Confidence Interval } \\
\hline & & & & & & Lower Bound & Upper Bound \\
\hline \multirow[t]{5}{*}{ Temperature oC } & \multirow[t]{3}{*}{ Winter } & Spring & $-5.120000^{*}$ & 666108 & .000 & -6.53209 & -3.70791 \\
\hline & & Summer & $-7.820000^{\star}$ & 666108 & .000 & -9.23209 & -6.40791 \\
\hline & & Autumn & $-4.900000^{*}$ & .666108 & .000 & -6.31209 & -3.48791 \\
\hline & Spring & Summer & $-2.700000^{*}$ & .666108 & .001 & -4.11209 & -1.28791 \\
\hline & Summer & Autumn & $2.920000^{*}$ & .666108 & .000 & 1.50791 & 4.33209 \\
\hline \multirow[t]{3}{*}{ Electrical conductivity $(\mu \mathrm{S} / \mathrm{cm})$} & Winter & Spring & $1781.200000^{*}$ & 439.522821 & .001 & 849.45324 & 2712.94676 \\
\hline & \multirow[t]{2}{*}{ Spring } & Summer & $-1974.400000^{*}$ & 439.522821 & .000 & -2906.14676 & -1042.65324 \\
\hline & & Autumn & $-2449.400000^{*}$ & 439.522821 & .000 & -3381.14676 & -1517.65324 \\
\hline Specific density & Spring & Autumn & $-.002400^{\star}$ & .000755 & .006 & -.00400 & -.00080 \\
\hline \multirow[t]{4}{*}{ Sulphate $\left(\mathrm{SO}_{4}^{-}\right)(\mathrm{mg} / \mathrm{l})$} & \multirow[t]{2}{*}{ Winter } & Summer & $758.800000^{*}$ & 134.573586 & .000 & 473.51674 & 1044.08326 \\
\hline & & Autumn & $595.400000^{*}$ & 134.573586 & .000 & 310.11674 & 880.68326 \\
\hline & \multirow[t]{2}{*}{ Spring } & Summer & $799.800000^{*}$ & 134.573586 & .000 & 514.51674 & 1085.08326 \\
\hline & & Autumn & $636.400000^{*}$ & 134.573586 & .000 & 351.11674 & 921.68326 \\
\hline \multirow[t]{4}{*}{ Nitrite $\left(\mathrm{NO}_{2}^{-}\right) \quad(\mathrm{mg} / \mathrm{l})$} & \multirow[t]{2}{*}{ Winter } & Spring & $.012200^{*}$ & .004921 & .025 & .00177 & .02263 \\
\hline & & Autumn & $-.035200^{*}$ & .004921 & .000 & -.04563 & -.02477 \\
\hline & Spring & Autumn & $-.047400^{*}$ & .004921 & .000 & -.05783 & -.03697 \\
\hline & Summer & Autumn & $-.043200^{*}$ & .004921 & .000 & -.05363 & -.03277 \\
\hline \multirow[t]{3}{*}{ Ammonia $\left(\mathrm{NH}_{3}\right)(\mathrm{mg} / \mathrm{l})$} & Winter & Summer & $-.074000^{\star}$ & .027148 & .015 & -.13155 & -.01645 \\
\hline & Spring & Summer & $-.076000^{*}$ & .027148 & .013 & -.13355 & -.01845 \\
\hline & Summer & Autumn & $.058000^{*}$ & .027148 & .048 & .00045 & .11555 \\
\hline \multirow[t]{3}{*}{ Chloride (mg/L) } & Winter & Summer & $-17191.400000^{*}$ & 2851.075920 & .000 & -23235.41095 & -11147.38905 \\
\hline & Spring & Summer & $-14854.800000^{*}$ & 2851.075920 & .000 & -20898.81095 & -8810.78905 \\
\hline & Summer & Autumn & $14183.000000^{*}$ & 2851.075920 & .000 & 8138.98905 & 20227.01095 \\
\hline
\end{tabular}




\begin{tabular}{|c|c|c|c|c|c|c|c|}
\hline \multirow[t]{3}{*}{ Total hardness $\left(\mathrm{CaCO}_{3} / \mathrm{l}\right)$} & Winter & Autumn & $-7516.000000^{*}$ & 1670.928335 & .000 & -11058.20983 & -3973.79017 \\
\hline & Spring & Autumn & $-9670.600000^{*}$ & 1670.928335 & .000 & -13212.80983 & -6128.39017 \\
\hline & Summer & Autumn & $-8058.800000^{*}$ & 1670.928335 & .000 & -11601.00983 & -4516.59017 \\
\hline \multirow[t]{4}{*}{ Total alkalinity $\left(\mathrm{CaCO}_{3} / \mathrm{l}\right)$} & \multirow[t]{2}{*}{ Winter } & Summer & $-357.400000^{*}$ & 62.819742 & .000 & -490.57190 & -224.22810 \\
\hline & & Autumn & $-284.800000^{*}$ & 62.819742 & .000 & -417.97190 & -151.62810 \\
\hline & \multirow[t]{2}{*}{ Spring } & Summer & $-305.600000^{*}$ & 62.819742 & .000 & -438.77190 & -172.42810 \\
\hline & & Autumn & $-233.000000^{*}$ & 62.819742 & .002 & -366.17190 & -99.82810 \\
\hline \multirow[t]{5}{*}{ Magnesium Hardness (mg/l) } & \multirow[t]{3}{*}{ Winter } & Spring & $-2837.800000^{*}$ & 882.270548 & .005 & -4708.13001 & -967.46999 \\
\hline & & Summer & $-4957.600000^{*}$ & 882.270548 & .000 & -6827.93001 & -3087.26999 \\
\hline & & Autumn & $-4792.800000^{*}$ & 882.270548 & .000 & -6663.13001 & -2922.46999 \\
\hline & \multirow[t]{2}{*}{ Spring } & Summer & $-2119.800000^{*}$ & 882.270548 & .029 & -3990.13001 & -249.46999 \\
\hline & & Autumn & $-1955.000000^{*}$ & 882.270548 & .042 & -3825.33001 & -84.66999 \\
\hline \multirow[t]{2}{*}{ Calcium Hardness (mg/l) } & \multirow[t]{2}{*}{ Winter } & Summer & $1576.200000^{*}$ & 531.986898 & .009 & 448.43816 & 2703.96184 \\
\hline & & Autumn & $1723.000000^{*}$ & 531.986898 & .005 & 595.23816 & 2850.76184 \\
\hline \multirow[t]{2}{*}{ Manganese $(\mathrm{Mn})(\mu \mathrm{gm} / \mathrm{l})$} & Winter & Autumn & $.003400^{\star}$ & .001428 & .030 & .00037 & .00643 \\
\hline & Spring & Autumn & $.004000^{*}$ & .001428 & .013 & .00097 & .00703 \\
\hline
\end{tabular}

*Significant at the 0.05 level.

\section{Conclusion}

Based on the results of descriptive and multivariate statistics analysis in the Dumate al-Jandal water lake, observation assessed the linkages between spatio-temporal variability and water quality using. Not all sampled parameters indicated significant spatio-temporal variability. Multivariate analysis and ANOVA even its among seasonal variation or between sites construed that the lake water quality is primarily influenced by agricultural runoff and their original water resources. On the basis of molar concentrations most of the variations in water quality are explained by the natural soluble salts, nonpoint source nutrients, and environmental inorganic pollutants. The present study, concluded that the chemical parameters of water lake led to bad quality of water lake which resulted from the interaction of some environmental component. However, the

higher and lower changes among the physical properties, such as temperature, $\mathrm{pH}, \mathrm{EC}$, turbidities, specific density and total dissolved solids in addition to chemical characteristics like the concentration of nitrate, nitrite, ammonia, phosphate, sulphate total hardness, calcium hardness, and magnesium hardness were observed are not suitable for growth of aquatic organisms of the ecosystem services and activities. Based on the recorded observation and concentrations, the lake water quality is under strong natural pressure. Therefore, water resources management are recommended to solve such acute problems for sustainably use to hit the intended objective. This research emphasized that were observed in terms of molar concentration a highest in spring and lowest in autumn and winter at all the sites. But lower levels were observed at other sites among the seasonal variation; the lowest values were recorded in the winter at all the sites. The overall values of Multivariate Test Using Wilks' Statistics showed a significant difference of concentrations between sites for each parameter. On the basis of molar concentrations, among the anions which have a major concentration in lake and heavy metals in terms of their cations were found in a minor and moderate concentrations.

\section{Conflict of Interests}

The authors declare that there is no conflict of interests regarding part 1 of the publication of this paper.

\section{Acknowledgements}

The author would like to acknowledge the support of Porf. Basher Jarrar - Jerash private unversity, Dr. Meshruf Alrwaylli - Aljouf university, Dr. Mohanad Sheraydeh- Jordan University of Science and Technology and Dr. Basel M. Eideh Quantitative Methods \& Information Systems Department - Kuwait University - Kuwait. 


\section{REFERENCES}

1. https://en.wikipedia.org/wiki/Dumat_al-Jandal.

2. Efflers S. W., Perkins M. G., Garofalo J. E., Roop R., Johnson D. and Nancy Auer (1988). Limnological analysis of Owasco lake for 1986. Upstate Freshwater Institute, Inc., Syracuse, New York.

3. Abood MM, Mohammed TA, Ghazali AH, Mahmud AR, Sidek (2009). Study and assessment for sedimentation models applied to impounding reservoirs. J. Eng. Appl. Sci. 4(2):152-160.

4. Asriningtyas V, Meesukko C, Gajaseni N, Voinov A (2005). Seasonal water flow trends in conjunction with phytoplankton biovolume in Phetchaburi River, Thailand. J. Sci. Resr. Chulalongkorn Univer. 30:161178.

5. Atobatele OE, Ugwumba OA (2008). Seasonal variation in the physicochemistry of a small tropical reservoir (Aiba Reservoir, Iwo, Osun, Nigeria). Afr. J. Biotechnol. 7(12):1962-1971.

6. Gregory Carey. 1998.Multivariate Analysis of Variance (MANOVA): I. Theory. http: //ibgwww .colorado.edu / carey /p7291dir/handouts /manova1.pdf. Accessed 16 November 2009.

7. Mardia, K.V.; J.T. Kent, J.M. Bibby. 1979. Multivariate Analysis. Academic Press.

8. V. Simeonov, P. Simeonova, and R.Tsitouridou, “Chemometric quality assessment of surface waters two case studies, "Chemical and Engineering Ecology,vol.11,no.6, pp.449-469, 2004.

9. K. P. Singh, A. Malik, D. Mohan, and S. Sinha, "Multivariate statistical techniques for thee valuation of spatial and temporal variations in water quality of Gomt iRiver (India): a case study," Water Research, vol.38, no.18,pp.3980-3992,2004.

10. Parsons T.R., Mait Y. and Laulli C.M. (1984). A manual of chemical and biological methods for seawater analysis. Pergamone press, Oxford.

11. APHA (1985). Standard Methods for the Examination of Water and Wastewater. 20 ${ }^{\text {th }}$ Ed. American Public Health Association, Washington, DC.

12. Chemometric Techniques for Quantitative Analysis 1st Edition, Kindle Edition by KRAMER. RICHARD (Author), 1998.

13. SPSS (version. 22) software was used to perform for a statistical analyses and their applications.

14. K. Van Der Gucht, K. Sabbe, L. De Meester et al., "Contrasting bacterio plankton community composition and seasonal

15. dynamics in two neighbouring hypertrophic fresh water lakes," Environmental Microbiology, vol.3, no.11, pp.680-690,2001.

16. A.H.Mahvi,J.Nouri, A.A. Babaei, and R.Nabizadeh,"Agricultural activities impact on ground water nitrate

17. pollution,"International Journal of Environmental Science and Technology, vol. 2,no.1,pp.41-47,2005.

18. Efflers S. W., Perkins M. G., Garofalo J. E., Roop R., Johnson D. and Nancy Auer (1988). Limnological analysis of Owasco lake for 1986. Upstate Freshwater Institute, Inc., Syracuse, New York. 
19. USEPA, Recent Recommended Water Quality Criteria, United States Environmental Protection Agency,2007.

20. Mustapha, Moshood \& S Omotoso, J. (2008). An assessment of the physico-chemical properties of Moro lake. African Journal of Applied Zoology and Environmental Biology. 7. 10.4314/ajazebvi1.41151.

21. H. P. Jarvie, B. A. Whitton, and C. Neal, "Nitrogen and phosphorus in east coast British rivers: speciation, sources and biological significance," Science of the Total Environment, vol.210211, pp.79-109,1998.

22. Ayalew W, Seyoum M, Vijverberg J, Eshetie D (2007). Seasonal variation in primary production of a large high altitude lakes (Lake Tana, Ethiopia): Effects of nutrient availability and water transparency. C. Springer science Business media B.V. P 197.

23. Ayoola SO, Kuton MP (2009). Seasonal variation in fish abundance and physicochemical parameters of Lagos Lagoon, Nigeria. Afr. J. Environ. Sci. Technol. 3(5):149-156. Bennett BL (1998).

24. Land Use Influences on Benthic Invertebrate Assemblages in Southern Appalachian agricultural streams. Virginia Polytechnic Institute and State University Blacksburg, Virginia pp. 38- 45.

25. Bellingham K (2004). Physicochemical Parameters of Natural Waters. Stevens Water Monitoring Systems, Inc.1 -800-452-5272. Retrieved on 25/4/2010, from http://www.stevenswater.com/articles/waterparameters.aspx Bhattarai KR, Shrestha BB, Lekhak HD (2008).

26. Water quality of Sundarijal Reservoir and its feeding streams in Kathmandu. Bhattarai, Sainik Awasiyaa Mahavidyalaya, Bhaktapur, Nepal. Sci. World 6(6):99-105. Chapman D (1996). Water Quality Assessments - A Guide to Use of Biota, Sediments and Water in Environmental Monitoring. (2nd edn.). ISBN 0419215905 (HB) 0419216006 (PB) Publ.

27. Hassan F. M., Kathim N. F. and Hussein, F. H. (2008). Effect of chemical and physical properties of river water in Shatt Al-Hilla on phytoplankton communities. E-Journal of Chemistry, 5: 323-330.

28. Simmons, G. M. and Reay, W. G. (1992). The phenomenon called submarine groundwater discharge. First International Conference on Ground Water Ecology, US Environmental Protection Agency. American Water Resource Association. 